\title{
Multistage-responsive nanocomplexes attenuate ulcerative colitis by improving the accumulation and distribution of oral nucleic acid drugs in the colon
}

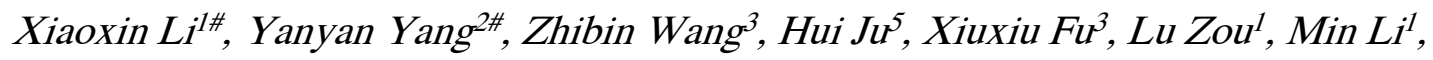 \\ Qianqian Xue ${ }^{1}$, Huibo $\mathrm{Ma}^{4}$, Yuanyuan Meng ${ }^{3}$, Liang Zhao ${ }^{3}$, Hongzhao Qi ${ }^{1, *}$, and Tao \\ $Y u^{1,3 *}$
}

1 Institute for translational medicine, The Affiliated Hospital of Qingdao University, College of Medicine, Qingdao University, Qingdao 266021, China;

2 Department of Immunology, Basic Medicine School, Qingdao University, Qingdao 266021, China;

3 Department of Cardiac Ultrasound, The Affiliated Hospital of Qingdao University, Qingdao 266000, China;

4 Department of Vascular Surgery, The Affiliated Hospital of Qingdao University, Qingdao 266000, China.

5 Department of Gastroenterology, The Affiliated Hospital of Qingdao University, Qingdao 266000, China.

\#These authors contributed equally to this paper.

* Corresponding author:

Tao Yu, e-mail: yutao0112@qdu.edu.cn;

Hongzhao Qi, e-mail: qihongzhao@qdu.edu.cn 


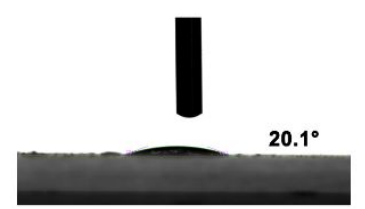

pH 7.0

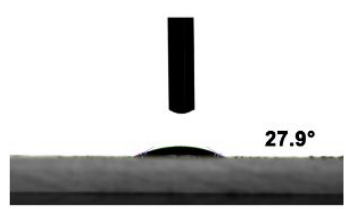

pH 5.0

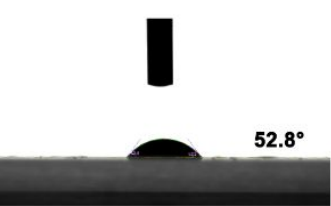

pH 3.0

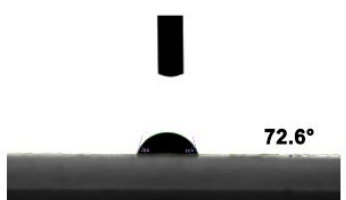

pH 1.0

Figure S1. The water contact angle of films respectively formed by MSNs that were placed in different $\mathrm{pH}$ solutions.

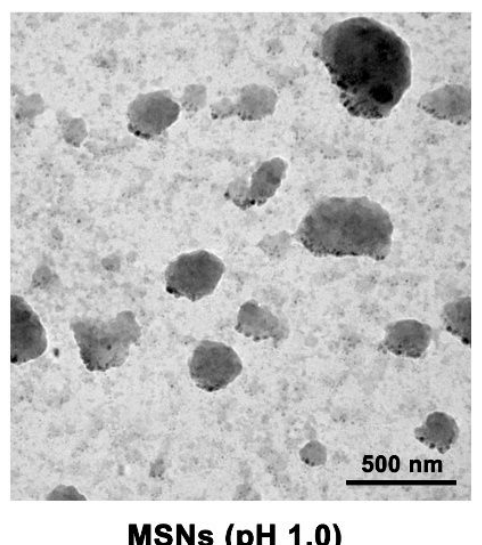

MSNs (pH 1.0)

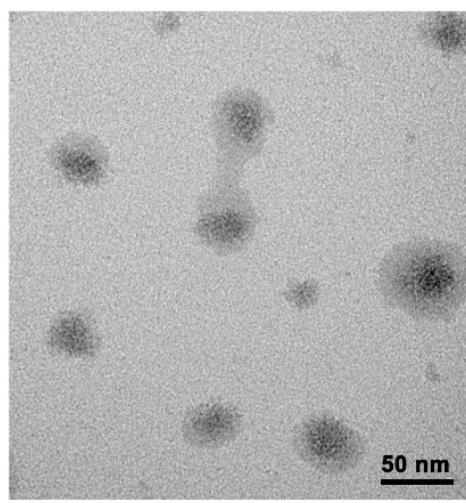

MSNs incubated with trypsin

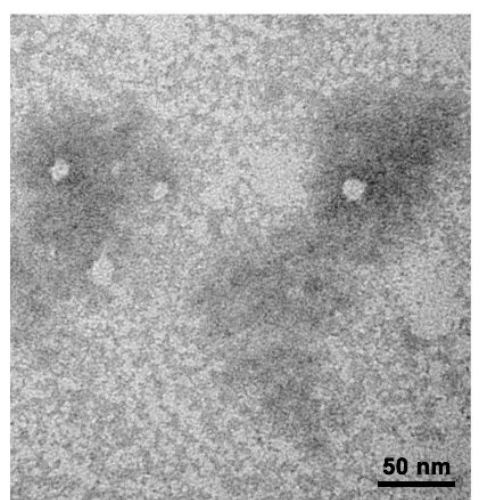

MSNs incubated with trypsin and mercaptoethanol

Figure S2. The representative TEM images of MSNs. Left: MSNs were placed in pH 1.0 solution; Middle: MSNs were incubated with trypsin $(10 \mu \mathrm{M})$ at $37^{\circ} \mathrm{C}$ for $4 \mathrm{~h}$; Right: MSNs were firstly incubated with trypsin $(10 \mu \mathrm{M})$ for $4 \mathrm{~h}$ at $37^{\circ} \mathrm{C}$ and then incubated with mercaptoethanol $(50 \mu \mathrm{M})$ for $4 \mathrm{~h}$ at $37^{\circ} \mathrm{C}$.

RAW264.7

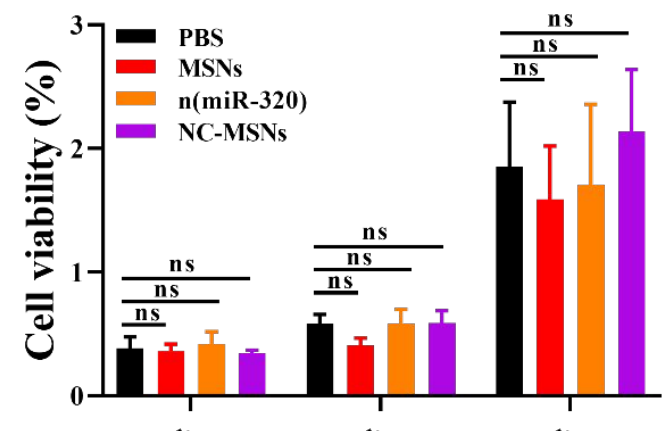

120
$2^{4 n}$
$4^{8 n}$
Caco-2

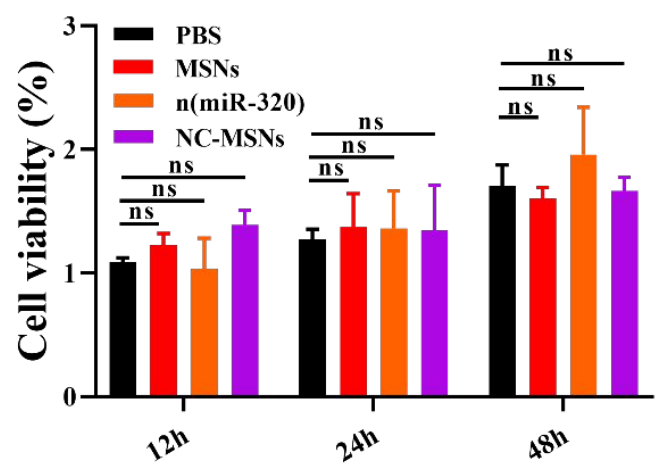


Figure S3. Cytotoxicity evaluation. MTT cell proliferation assay was used to evaluate the potential toxicity of MSNs, n(miR-320), and NC-MSNs in RAW264.7 and Caco-2 cells. All values are means \pm SD and ${ }^{\text {nsP }}>0.05$.
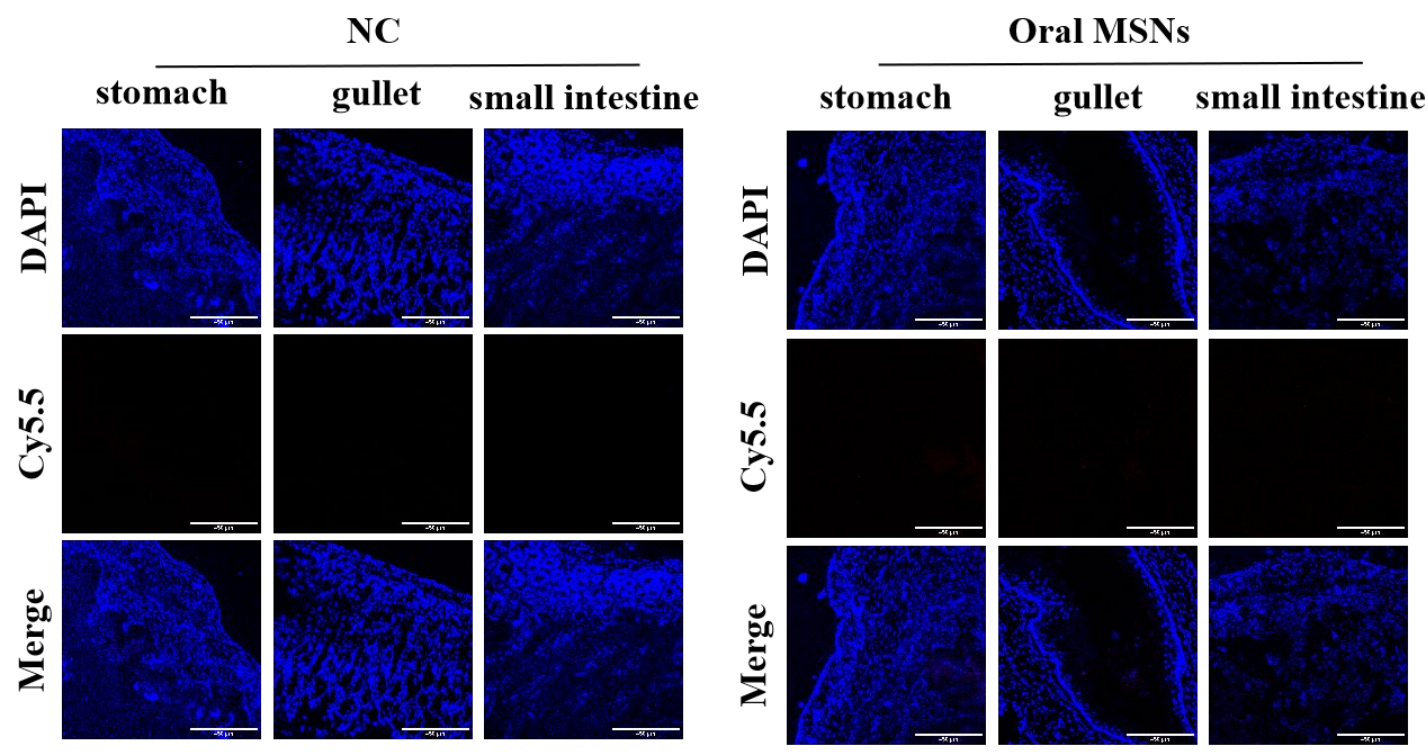

Figure S4. The distribution of Cy5.5-labeled miR-320 in the gastrointestinal tissue section was evaluated using a confocal fluorescence microscope; blue represented cell nucleus and red represented miR-320. The scale bar was $400 \mu \mathrm{m}$.
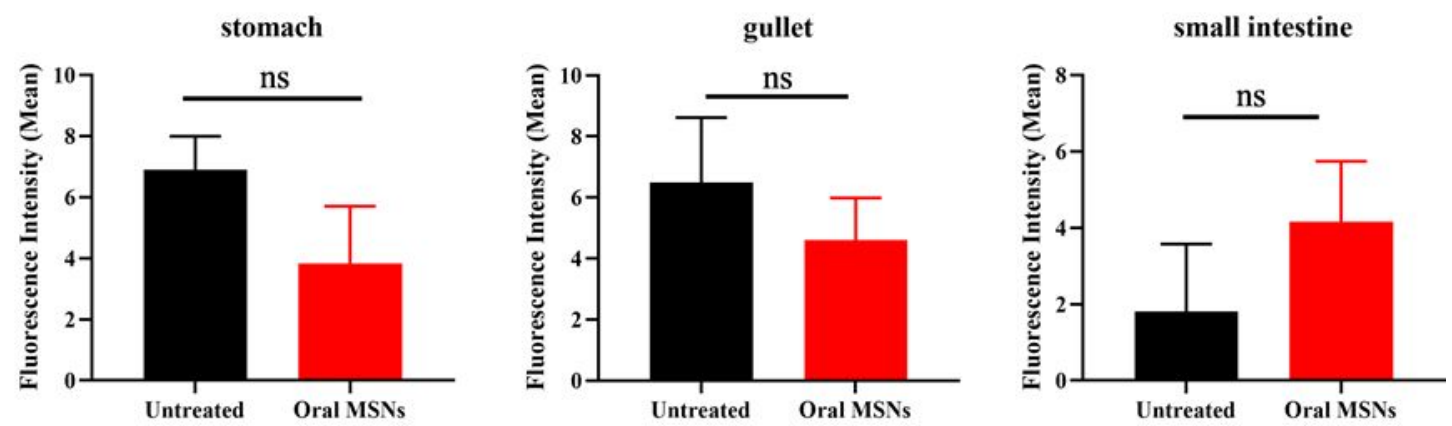

Figure S5. Quantification of fluorescence intensity in the gastrointestinal tissue section by ImageJ software. 

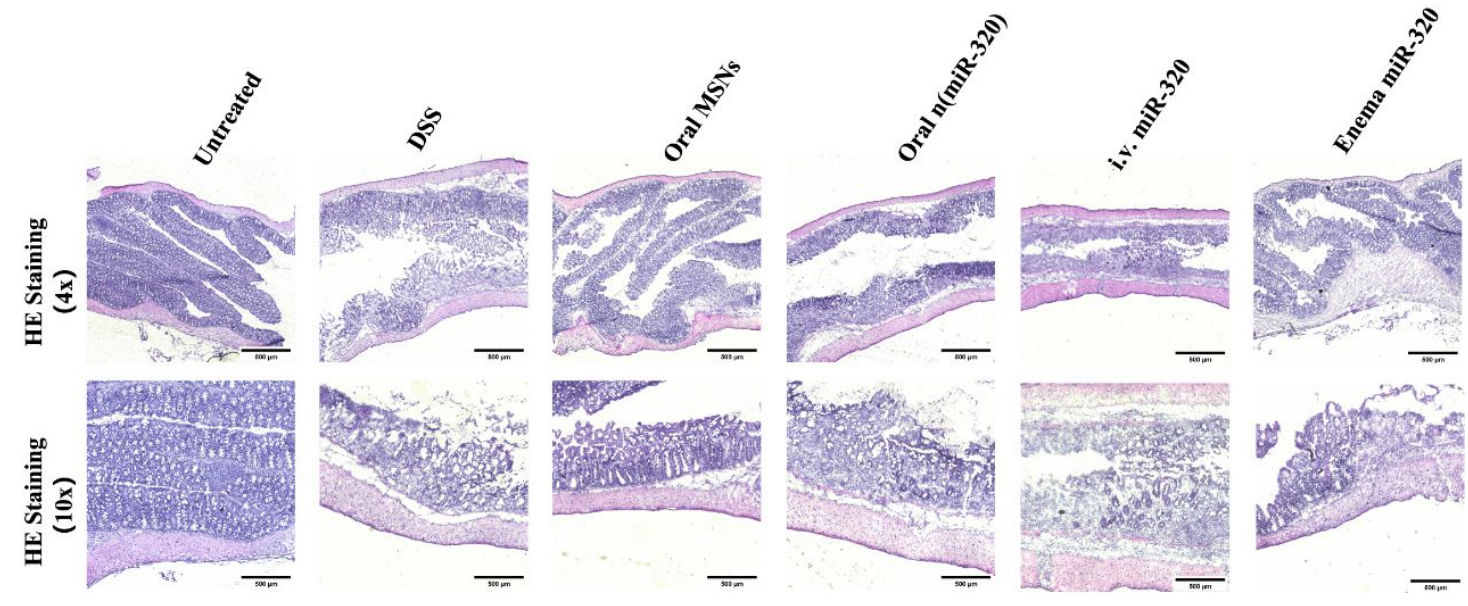

Figure S6. Longitudinal sections of the distal colon were stained with H\&E on day 7. Scale bars were $500 \mu \mathrm{m}$. 